\title{
LDA and GGA Investigations of Some Ground-State Properties and the Compton Profile of Copper with the All-Electron MAPW Method
}

\author{
Helmut Bross \\ Arnold Sommerfeld Center, University of Munich, Theresienstraße. 37 IV, 80333 Munich, Germany \\ Correspondence should be addressed to Helmut Bross, helmut.bross@physik.uni-muenchen.de \\ Received 26 September 2011; Accepted 13 October 2011 \\ Academic Editors: S. B. Dugdale and J. E. Lowther \\ Copyright (C) 2012 Helmut Bross. This is an open access article distributed under the Creative Commons Attribution License, \\ which permits unrestricted use, distribution, and reproduction in any medium, provided the original work is properly cited.

\begin{abstract}
The electronic structure is self-consistently evaluated both in the local-density and the generalized-gradient approximations. The convergence with respect to the intrinsic parameters of the modified augmented plane-wave scheme is thoroughly investigated. Besides the Ar-core, the less-extended $\mathrm{Ne}$-core and the most appropriate exchange-correlations functionals are considered. It is studied how some characteristic ground-state properties and the Compton profiles depend on these choices. Apart from the long wave limit of the Compton profiles, most of the other properties are found in good agreement with the experimental facts.
\end{abstract}

\section{Introduction}

The electronic structure of the noble metal $\mathrm{Cu}$ has been investigated extensively in the last four decades and reasonable agreement between theory and experiment was found. In most theoretical examinations the core state is chosen to be of Ar type which considerably simplifies the numerical work as the band structure scheme has only to be applied to the $4 \mathrm{~s}$ and $3 \mathrm{~d}$ electrons. However, the wave functions of the $3 \mathrm{~s}$ and $3 \mathrm{p}$ electrons do not completely vanish at the surface of the sphere lying fully within the atomic cell with the consequence that these outer core electrons dynamically interact with the valence electrons. (Typically their wave functions differ by the factors 80 and 37 from the corresponding values of the $4 \mathrm{~s}$ and $4 \mathrm{p}$ functions, resp.). It is interesting to examine to what extent the agreement is improved not only by including the outer core electrons in the band structure calculations but also by the use of more advanced theoretical schemes combined with the use of more powerful computers which allow a considerable increase of various parameters inherent in the computational schemes.

The outline of the paper is as follows. In Section 2 we start with a review of the modified augmented plane wave (MAPW) scheme $[1,2]$. Then we will confine ourselves to some specific features as well as the proper choice of some intrinsic parameters of the MAPW scheme are described. Section 3 is devoted to the evaluation of the most important ground-state properties within the frame work of the local density approximation (LDA) and the generalized gradient approximation (GGA). In both approximation, the most appropriate exchange-correlation functionals [35] are applied. Section 4 resumes former investigations of the Compton profiles (CP) by the author [6] on a far more refined level nowadays possible. The MAPW scheme turns out to be best suited to meet with this problem without any truncations. At first we study the influence of the spherically averaged electron moment density (EMD) on the Compton profiles. Because it can be done quite efficiently it allows extensive investigations of different models and assumptions. In the following subsections, the directional Compton profiles are investigated. A critical comparison with previous Kohn-Korringa-Rostocker (KKR) studies by Sakurai et al. [7] concludes the work.

\section{The Electronic Structure}

2.1. Basic Concepts of the MAPW-Method. Since the MAPW method has received ample documentation elsewhere $[1,2]$, we will confine ourselves to reviewing only its most important features. As to the original APW-method [8], the trial 
functions outside the atomic spheres are approximated by a superposition of plane waves

$$
\chi_{\vec{k}}(\vec{r})=\sum_{\vec{K}} v(\vec{K}) e^{i(\vec{k}+\vec{K}) \cdot \vec{r}}
$$

where the summation over the reciprocal fcc lattice vectors is restricted by the inequality

$$
(\vec{k}+\vec{K})^{2} \leq \text { QMXSQ }\left(\frac{2 \pi}{a}\right)^{2} .
$$

QMXSQ is a dimensionless magnitude which controls the number of plane waves $N_{\mathrm{pw}}$. This choice of the reciprocal vectors guarantees the full point group symmetry of the Bloch energies $E_{\vec{k}}$ at any $\vec{k}$ in the Brillouin zone. The optimal choice of QMXSQ is discussed below. To comprehend the form of the trial functions within the atomic spheres, the right-hand side of (1) is rewritten by use of the plane waves expansion in spherical coordinates with origin at the central atomic polyhedron

$$
\begin{aligned}
\sum_{\vec{K}} v(\vec{K}) e^{i(\vec{k}+\vec{K}) \cdot \vec{r}} \\
=4 \pi \sum_{l=0}^{\infty} \sum_{m=-l}^{m=l}(-1)^{m} i^{l} Y_{l, m}\left(\vec{r}^{0}\right) \\
\quad \times \sum_{\vec{K}} v(\vec{K}) Y_{l,-m}\left((\vec{k}+\vec{K})^{0}\right) j_{l}(|\vec{k}+\vec{K}| r) .
\end{aligned}
$$

$Y_{l, m}$ are normalized spherical Harmonics and $j_{l}$ are spherical Bessel functions of first kind. Unit vectors are denoted by the superscript 0 . It is clear that the terms with small values of $l$ do not properly describe the influence of the crystal potential near the nuclei. Following Slater [8], we substitute the spherical Bessel functions for $l \leq L$ by a superposition of properly chosen linear combination of radial functions $R_{s l}(r)$. The first index $s$ counts the elements of the set. This augmentation idea leads finally inside the central atomic sphere to the Ansatz

$$
\begin{aligned}
\chi_{\vec{k}}(\vec{r})= & 4 \pi \sum_{l=0}^{L} \sum_{m=-l}^{m=l}(-1)^{m} i^{l} Y_{l, m}\left(\vec{r}^{0}\right) \sum_{s} A_{s l m} R_{s l}(r) \\
& +4 \pi \sum_{l=L+1}^{\infty} \sum_{m=-l}^{m=l}(-1)^{m} i^{l} Y_{l, m}\left(\vec{r}^{0}\right) \\
& \times \sum_{\vec{K}} v(\vec{K}) Y_{l,-m}\left((\vec{k}+\vec{K})^{0}\right) j_{l}(|\vec{k}+\vec{K}| r)
\end{aligned}
$$

which alternatively may be rewritten as

$$
\begin{aligned}
\chi_{\vec{k}}(\vec{r})=\sum_{\vec{K}} v(\vec{K}) e^{i(\vec{k}+\vec{K}) \cdot \vec{r}} \\
+4 \pi \sum_{l=0}^{L} \sum_{m=-l}^{m=l}(-1)^{m} i^{l} Y_{l, m}\left(\vec{r}^{0}\right) \\
\times\left[\sum_{s} A_{s l m} R_{s l}(r)-\sum_{\vec{K}} v(\vec{K}) Y_{l,-m}\right. \\
\left.\times\left((\vec{k}+\vec{K})^{0}\right) j_{l}(|\vec{k}+\vec{K}| r)\right]
\end{aligned}
$$

by use of the plane waves expansion.

Summarizing, each trial function $\chi$ of the MAPW scheme is a linear combination of plane waves which are, for any angular quantum number $l \leq L$, augmented by the square bracket of (5) within the atomic spheres. The radial functions $R_{s l}$ are solutions of the spherical radial differential equation with properly spherically averaged crystal potential within the atomic spheres. For each value of $l \leq L$ a set of $R_{s l}$ counted by the first index $1 \leq s \leq N R(l)$ is used. $R_{s l}$ is generated by requiring a given value of the logarithmic derivative $\left(d R_{s l} / d r\right) / R_{s l}$ on the surface of the atomic sphere $r_{\mathrm{APW}}$. It turned out to be mostly sufficient to choose these logarithmic derivatives to be either 1 or -1 .

In contrast to other versions of APW each trial function as well as its gradient are made continuous everywhere in the atomic polyhedron by requiring that the corresponding square brackets of (5) vanish for $r=r_{\mathrm{APW}}$

$$
\begin{array}{r}
\sum_{s} A_{s l m} R_{s l}(r)=\sum_{\vec{K}} v(\vec{K}) Y_{l,-m}\left((\vec{k}+\vec{K})^{0}\right) j_{l}(|\vec{k}+\vec{K}| r) \\
\forall(-l \leq m \leq l, 0 \leq l \leq L)
\end{array}
$$

and the analogous equation with the first derivative with respect to $r$. In the variational procedure which makes the expectation value of the crystal Hamiltonian $H$ extremal, these equations play the role of additional constraints with the consequence that a general Hermitian eigenvalue problem of rank $N_{\mathrm{PW}}+3 \sum_{l=0}^{L} N R(l)$ has to be solved. For a given value of $\vec{k}$ it gives Bloch states with energies $E_{n \vec{k}}$ and wave functions $\langle n \vec{k} \mid \vec{r}\rangle$ where $n \leq N_{\mathrm{PW}}+\sum_{l=0}^{L}\left(N R_{l}-2\right)$. Approximately only a quarter of them is useful in further applications.

With respect to all-electron investigations the present schema has the advantage to allow the incorporation of the cores in a simple and rigid way. As core wave functions are completely localised within the atomic spheres, the lefthand side of (4) vanishes even when the coefficients $A_{s l m}$ are nonzero. Consequently no plane waves exist outside the atomic spheres. By use of the radial differential equation, it can be shown that the core and valence electron wavefunctions are strictly orthogonal on each other.

A critical comparison in a recent paper [9] had the result that the MAPW scheme guarantees a higher accuracy than 
the APW schemes mostly used by other groups especially with regard to the EMD and CP.

2.2. Evaluation of the Electronic Structure of $\mathrm{Cu}$. The valence electrons of $\mathrm{Cu}$ are of s-, p-, and d-type. Due to the dominant role of the d-electrons, we choose $R_{1, l=2}$ to be almost atomlike, for example, both $R_{1,2}$ and $d R_{1,2} / d r$ are made small on the surface of the atomic sphere by a properly chosen value of the energy in the radial differential equation. All other radial functions are generated with the recipe of Section 2.1. The electronic structure was self-consistently determined by use of the MAPW formalism yielding Bloch functions $\langle\vec{r} \mid n \vec{k}\rangle$ which due to their continuity within the atomic polyhedron are especially suited for the evaluation of the electron momentum density (EMD). For details we refer to previous investigations [10-13]. Complete self-consistent calculations for both the $[\mathrm{Ne}]$ and $[\mathrm{Ar}]$ cores are performed which treat 19 or 11 electrons, respectively, by the band structure scheme. In the present investigation it proves to be quite advantageous that, different from other schemes, the MAPW method yields Bloch states extending over an energy range of 8 Ry which are strictly orthogonal to each other.

As a peculiarity of the MAPW formalism in the angular momentum decomposition of the wave function within the APW sphere only 5 different properly chosen radial functions are sufficient for $l \leq 2$.

The density of the electrons $\rho(\vec{r})$ obtained from the occupied states is, as a consequence of the MAPW ansatz, a symmetric combination of plane waves everywhere and angular-dependent contributions within the atomic spheres. We have found it to be sufficiently accurate in the case of $\mathrm{Cu}$ to evaluate the latter contributions along 12 specially chosen directions [14-18] in the $1 / 48$ of the unit sphere and to construct the crystal potential in the warped muffintin approximation by averaging over all angular-dependent contributions of these directions. In the sense of Fehlner et al. $[15,16]$ this set of directions allows the exact evaluation of the expansion coefficients of all Kubic harmonics (KHs) up to $l=16$. Outside the APW sphere the Coulomb potential is obtained without any further simplification, whereas the exchange and correlation as well as the Lam-Platzman(LP) [19] corrections could be treated in any accuracy wanted by use of a suitably chosen fine mesh of $\vec{r}$ points [12]. Consistent with the other intrinsic inaccuracies 916 points in the irreducible wedge are considered.

The Brillouin zone (BZ) integrations over all occupied states yielding the electron density $\rho(\vec{r})$, the Fermi energy $E_{F}$, the total energy $E_{\text {tot }}$, and the EMD are approximated by a sum over $M G(M G+1)(M G+2) / 12$ points properly chosen in the irreducible wedge [20-23]. These points constitute a simple cubic lattice of length $(1 / 2 \mathrm{MG})(2 \pi / a)$. The characteristic ground-state properties are calculated by use of the LDA functional [3] and of the GGA functionals $[4,5,24,25]$.

Extensive numerical investigations with QMXSQ extending up to 24.0 and MG up to 40 have been performed to analyze how these intrinsic parameters influence the groundstate properties. We have found that the asymptotic behavior, that is, MG $\rightarrow \infty$ and QMXSQ $\rightarrow \infty$, of the total energy minimized in the LDA scheme is well-described by the expression

$$
E_{\mathrm{tot}}=P_{1}+\frac{P_{2}}{\mathrm{MG}^{\nu}}+P_{3} e^{-\mu \mathrm{QMXSQ}} .
$$

Because of the nonlinear parameters $\mu$ and $\nu$, the fitting procedure is divided into two steps: (1) For fixed values of $\mu$ and $\nu$ the parameters $P_{1}, P_{2}$, and $P_{3}$ have been determined by the least-squares method, and (2) the minimum of the sum of the squared residuals over the $(\mu, \nu)$-plane gives the optimal values of the nonlinear parameters. In the case of the $[\mathrm{Ne}]$ core, LDA and $a=6.8312171 \mathrm{Bohr} P_{1}=$ $-3275.9028707 \mathrm{Ry}, P_{2}=-1.220 \pm 0.160 \mathrm{Ry}, P_{3}=13.002 \pm$ $0.443 \mathrm{Ry}, v=2.726 \pm 0.044$, and $\mu=0.58658 \pm 0.00211$ are found. The positive sign of $P_{3}$ follows from the minimal property of $E_{\text {tot }}$, whereas the negative sign of $P_{2}$ is probably caused by the partitioning of the $\vec{k}$ space relative to the Fermi surface. According to this formula the $E_{\text {tot }}$ is obtained with an accuracy of $2 \mathrm{mRy}$ provided QMXSQ is chosen larger than 16.4. Then about 75 plane waves constitute a Bloch function. This number certainly is a lower limit for other plane wavelike schemes. For a proper rate of the magnitude of this energy a comparison with the zero-point energy of $\mathrm{Cu}$ is helpful which within the Debye approximation amounts to $2.66 \mathrm{mRy}$ (Debye temperature $\Theta=336 \mathrm{~K}$ and atomic volume $\left.V=79.70 \mathrm{Bohr}^{3}\right)$. With regard to the evaluation of the Compton profiles we have chosen the relatively large value $\mathrm{MG}=40$. The corresponding $\vec{k}$-grid contains 5740 points within the irreducible wedge and gives the total energy $E_{\text {tot }}$ and the Fermi energy $E_{F}$ with an accuracy of $50 \mu$ Ry and $58 \mu \mathrm{Ry}$, respectively.

This choice of the parameters together with the use of more elaborate exchange-correlation functionals guarantees results which can be considered as best obtainable approximation to the LDA at least in the warped-muffin-tin approximations. Remaining differences to the experimental results must be ascribed to many-body effects not properly approximated by LDA or GGA. Finally a useful remark: as the correction terms in (7) have opposite sign, their total contribution can be made zero by a proper choice of both MG and QMXSQ, for example, MG $=20$ requires $\mathrm{QMXSQ}=17.96$. Then $P_{1}$ is a good approximation to the asymptotic value of $E_{\text {tot }}$.

\section{Some Ground-State Properties}

Self-consistent calculations both in LDA and GGA were performed for up to 7 different lattice constants in the range $[6.48,7.10]$ a.u. in the fcc-structure. We have found that the total energy as function of the volume can be approximated with an absolute error of $10 \mu \mathrm{Ry}$ or less by the Murnagham expression [26]

$$
E_{\mathrm{tot}}=p_{1}+p_{2} V+p_{3} V^{1-\gamma}
$$

with the four adjustable parameters $p_{1}, p_{2}, p_{3}$, and $\gamma$. It describes a wide variety of behaviors: (i) $\gamma<0$ yields a more or less strong decrease at small values of $\mathrm{V}$ and 
(ii) $\gamma=3$ gives a symmetric energy curve around a certain minimum. The volume $V_{0}$, the isothermal bulk modulus $B_{0}$, and the total energy $E_{\text {tot, } 0}$ at the equilibrium configuration are determined by

$$
\begin{gathered}
V_{0}=\left[(\gamma-1) p_{3} / p_{2}\right]^{1 / \gamma}, \\
B_{0}=\gamma p_{2}, \quad E_{\text {tot }, 0}=p_{1}+p_{2} V_{0}+p_{3} V_{0}^{1-\gamma} .
\end{gathered}
$$

The nonlinear parameter $\gamma$ in the Murnagham expression numerically coincides with the pressure derivative of the bulk modulus $d B / d p$. As the latter is the third derivative of the total energy, a proper fit to the Murnagham expression requires energies $E_{\mathrm{tot}}$ with high intrinsic accuracy, say $1 \mu \mathrm{Ry}$, in the case of $\mathrm{Cu}$. As displayed in the case of $\mathrm{Al}$ [12] the energy-volume dependence is almost rigidly shifted upwards in accord with (7).

In Table 1 the result of the fit for the different LDA and GGA functionals and for both core models are listed. The corresponding experimental values are given in the last row. The lattices constant at $T=0 \mathrm{~K}$ was derived by use of the linear expansivity measured by Himmler et al. [27] using its value at $18^{\circ} \mathrm{C}$ [28]. The bulk modulus is taken from ultrasonic experiments at $T=4.2 \mathrm{~K}[29] . \gamma=d B / d p$ is a mean value of different measurements according to [30].

As in previous investigations [11], the GGA overestimates the equilibrium lattice constant $a_{0}$, whereas LDA underestimates it. Band structure calculations with the smaller core $[\mathrm{Ne}]$ shift $a_{0}$ closer to the experimental values. Compared to other theoretical investigations [4, 32], which mostly underestimate $a_{0}$ by $2 \%$, a considerable progress is achieved. The specific choice of the core has only a slight influence on the value of the bulk modulus $B$. Again LDA and GGA approach the experimental value from different sides where in LDA the difference is slightly smaller than in GGA, apart from the [Ar]pbe case which seems to be exceptional. In accord with the extremal principle $E_{\text {tot }}$ assumes in the [Ne] configuration a smaller value than in the corresponding [Ar] configuration; in LDA this difference is rather small. With regard to the volume dependence of the $B$ described by the Murnagham parameter $\gamma$ there is no unique behavior which is worth to be further discussed due the experimental uncertainty. In general the ground-state properties listed in Table 1 do not allow to favour one of the GGA functionals.

The Fermi surface of $\mathrm{Cu}$ consists of a distorted sphere at $\Gamma$ and is multiply connected at $\mathrm{L}$ by necks. Of special interest are the extremal orbits: the belly orbits $\mathrm{B}[100], \mathrm{B}[111]$, the dog's bone $\mathrm{DB}[110]$, and the rosette $\mathrm{R}[100]$. For further details see the review article [33]. In Tables 2 and 3 we present extremal areas $A$ and their dilatation dependence $d \ln A / d \ln V$ evaluated for different models and $a=3.6030 \AA$ $=6.80868$ Bohr, the experimental lattice constant at zero temperature. It is remarkable how close the results are found with different exchange-correlation functionals and with both choices of the core. For comparison experimental values of $A$ and of $d \ln A / d \ln V$ obtained by high precision de Haas van Alphen experiments $[34,35]$ are given in the last column. Apart from the neck orbit they differ from corresponding theoretical values by less than $1 \%$ that means
TABLE 1: Ground-state magnitudes for different exchange correlation functionals and both choices of the core. $5740 \vec{k}$ points within the irreducible wedge, QMXSQ $=15.5$ a.u. vwn: LDA functionals according Vosko et al. [3], pbe: GGA functionals according Perdew et al. [5], pw91: GGA functionals according Perdew et al. [4], and KKR: KKR results [31].

\begin{tabular}{lcccc}
\hline & $a_{0}(\mathrm{Bohr})$ & $E_{\text {tot }, 0}(\mathrm{Ryd})$ & $B(\mathrm{GPa})$ & $\gamma$ \\
\hline$[$ Ar]vwn & 6.7147 & -3275.901665 & 168.48 & 4.37 \\
[Ne]vwn & 6.7158 & -3275.902062 & 170.14 & 5.00 \\
[Ar]pbe & 7.0450 & -3280.834029 & 88.30 & 8.59 \\
[Ne]pbe & 6.9195 & -3280.844962 & 116.12 & 4.80 \\
[Ar]pw91 & 7.0394 & -3281.643558 & 106.62 & 6.11 \\
[Ne]pw91 & 6.9103 & -3281.654645 & 117.89 & 4.80 \\
KKR & 6.76 & -3275.768 & 155 & \\
exp & 6.8087 & & 142.03 & 4.9 \\
\hline
\end{tabular}

that the topology of the Fermi surface is quite insensitive to details of the potential. The relatively large error in the neck result is due to the fact that the extension of this orbit sensitively depends on the locations of the state $L_{2}^{\prime}$ relative to the Fermi level. A shift of approximately $8 \mathrm{mRy}$ would completely reduce this deficiency. Interestingly, a recent study [36] showed that a similar order of magnitude of the shift needed to be applied to calculations of the Fermi surface of Ag. Compared to the FLAPW investigations [37] which give the extended orbits with errors up to $8 \%$ both in LDA and SIC a considerable improvement is achieved. As the authors of this investigation concede the extremely small area of the neck orbit is a distinct disadvantage of the SIC approximation.

Using the Fermi surface radii along 48 suitably chosen directions on the unit sphere the coefficients of the expansion in symmetrized combinations of plane waves as defined in [39] have been evaluated by use of a singular value decomposition. The result listed in Table 4 confirms the previous results that the shape of the Fermi surface only weakly depends on the choice of the core and of the exchange correlation functionals. It is not surprising that there does not exist the faintest similarity with the values derived by Coleridge and Templeton $[34,40]$ from their high-precision data as their system of linear equations involved in the least-squares fitting procedure is not well conditioned. The preceding results are further supported by the fact that the energetic locations of some special states only weakly depend on the various approaches apart from the widths of the $3 \mathrm{~s}$ and $3 \mathrm{p}$ core states which are 0.0017 and $0.0033 \mathrm{Ryd}$, respectively, in the case of the $[\mathrm{Ne}]$-core.

Besides the total energy the charge density is the fundamental quantity of the density-functional formalism. Fortunately, it can directly be measured by the form factors of X-ray scattering or by $\gamma$-ray diffractometry. In Table 5 theoretical values obtained for different models with the lattice constant 3.614924 $\AA$ which is close to that of the room temperature value [28] are compared with measurements by Schneider et al. [41] and Temkin et al. [42]. Again we find that this quantity is quite insensitive to the special model 
TABLE 2: Extremal areas of cross-sections of the Fermi surface in units of the free electron cross section evaluated for $a=3.6030 \AA$.

\begin{tabular}{lccccccc}
\hline & {$[$ Ar]vwn } & {$[$ Ne]vwn } & {$[$ Ar]pbe } & [Ne]pbe & [Ar]pw91 & {$[\mathrm{Ne}] \mathrm{pw91}$} & exp. \\
\hline$\langle 100\rangle$ belly & .97320 & .97318 & .97226 & .97320 & .97285 & .97289 & .98062 \\
$\langle 111\rangle$ belly & .94309 & .94350 & .94268 & .94309 & .94337 & .94398 & .94983 \\
$\langle 111\rangle$ neck & .04032 & .03997 & .04143 & .04032 & .04035 & .03977 & .03555 \\
$\langle 110\rangle$ dogsbone & .40658 & .40684 & .40613 & .40658 & .40670 & .40711 & .41045 \\
$\langle 100\rangle$ rosette & .40418 & .40410 & .40362 & .40418 & .40392 & .40386 & .40241 \\
\hline
\end{tabular}

TABLE 3: Dilatation dependence of the cross-section $d \ln A / d \ln V$ evaluated for $a=3.6030 \AA$.

\begin{tabular}{lccccccc}
\hline & {$[\mathrm{Ar}] \mathrm{vwn}$} & {$[\mathrm{Ne}] \mathrm{vwn}$} & {$[\mathrm{Ar}] \mathrm{pbe}$} & {$[\mathrm{Ne}] \mathrm{pbe}$} & {$[\mathrm{Ar}] \mathrm{pw} 91$} & {$[\mathrm{Ne}] \mathrm{pw91}$} & exp. \\
\hline$\langle 100\rangle$ belly & -.6422 & -.6417 & -.6429 & -.6422 & -.6418 & -.6411 & -.628 \\
$\langle 111\rangle$ belly & -.6085 & -.6102 & -.6098 & -.6085 & -.6092 & -.6112 & -.598 \\
$\langle 111\rangle$ neck & -2.3641 & -2.3555 & -2.2763 & -2.3641 & -2.3562 & -2.3521 & -2.81 \\
$\langle 110\rangle$ dogsbone & -.5641 & -.5664 & -.5668 & -.5641 & -.5653 & -.5682 & -.574 \\
$\langle 100\rangle$ rosette & -.6426 & -.6404 & -.6452 & -.6426 & -.6418 & -.6390 & -.628 \\
\hline
\end{tabular}

TABle 4: Coefficients for seven term fits of the Fermi surface for different exchange-correlation functionals and both choices of the core. $5740 \vec{k}$ points within the irreducible wedge, QMXSQ = 15.5 a.u. Notation following Halse [38]. Last column: root mean of sum of squared energy residua.

\begin{tabular}{lcccccccc}
\hline & $C_{0}$ & $C_{200}$ & $C_{211}$ & $C_{220}$ & $C_{310}$ & $C_{222}$ & $C_{321}$ \\
\hline$[$ Ar]vwn & 5.17291 & 0.21625 & 0.30844 & 0.08518 & -0.01063 & -0.04044 & 0.00307 & 0.00046 \\
{$[\mathrm{Ne}]$ vwn } & 5.20912 & 0.21821 & 0.31581 & 0.08599 & -0.01007 & -0.03993 & 0.00308 & 0.00046 \\
{$[$ Ar]pbe } & 5.47841 & 0.23231 & 0.36842 & 0.09068 & -0.00397 & -0.03133 & 0.00276 & 0.00037 \\
{$[$ Ne]pbe } & 5.46965 & 0.23174 & 0.36651 & 0.09022 & -0.00391 & -0.03073 & 0.00270 & 0.00036 \\
{$[$ Ar]pw91 } & 5.20168 & 0.21776 & 0.31439 & 0.08597 & -0.01026 & -0.04019 & 0.00308 & 0.00046 \\
{$[$ Ne]pw91 } & 5.26010 & 0.22094 & 0.32623 & 0.08729 & -0.00934 & -0.03940 & 0.00311 & 0.00046 \\
\hline
\end{tabular}

TABLE 5: Form factors for different exchange- correlation functionals and both choices of the core. $5740 \vec{k}$ points within the irreducible wedge and QMXSQ = 15.5 a.u. (a) experimental values at $T=50 \mathrm{~K}$ (b); experimental values at room temperature.

\begin{tabular}{lcccccccc}
\hline & {$[$ Ar]vwn } & {$[\mathrm{Ne}] \mathrm{vwn}$} & {$[$ Ar]pbe } & {$[\mathrm{Ne}] \mathrm{pbe}$} & {$[$ Ar]pw91 } & {$[\mathrm{Nr}] \mathrm{pw91}$} & $(\mathrm{a})$ & $(\mathrm{b})$ \\
\hline$(111)$ & 21.685 & 21.603 & 21.700 & 21.622 & 21.700 & 21.620 & $21.51(5)$ & $21.93(14)$ \\
$(200)$ & 20.477 & 20.415 & 20.492 & 20.432 & 20.492 & 20.431 & $20.22(4)$ & $20.36(14)$ \\
$(220)$ & 16.760 & 16.747 & 16.770 & 16.757 & 16.772 & 16.759 & $16.45(5)$ & $16.70(15)$ \\
$(311)$ & 14.778 & 14.777 & 14.785 & 14.783 & 14.788 & 14.786 & $14.54(4)$ & $14.71(16)$ \\
$(222)$ & 14.240 & 14.241 & 14.246 & 14.246 & 14.249 & 14.249 & $14.07(5)$ & $14.18(16)$ \\
$(400)$ & 12.460 & 12.464 & 12.462 & 12.465 & 12.465 & 12.468 & $12.29(6)$ & $12.33(19)$ \\
$(420)$ & 11.165 & 11.167 & 11.164 & 11.166 & 11.167 & 11.169 & $11.02(6)$ & $10.08(6)$ \\
$(422)$ & 10.191 & 10.193 & 10.187 & 10.189 & 10.191 & 10.192 & $10.69(6)$ \\
$(333)$ & 9.609 & 9.611 & 9.604 & 9.606 & 9.608 & 9.610 & $9.49(6)$ \\
$(511)$ & 9.611 & 9.612 & 9.606 & 9.607 & 9.609 & 9.611 & $9.53(6)$
\end{tabular}

which is certainly due to the fact that the charge density is globally controlled by the requirement of charge neutrality. It is within the experimental margin that the data by Schneider slightly favour the model with the $[\mathrm{Ne}]$ core, whereas those of Temkin favour more the other core choice.

The cyclotron masses also turn out to be rather insensitive to the exchange-correlation functionals and the choice of the core. Typical values are 1.347, 1.404, 0.3931,
-1.210 , and -1.278 , for the $\langle 100\rangle$ belly, $\langle 111\rangle$ belly, $\langle 111\rangle$ neck, $\langle 110\rangle$ dogsbone, and the $\langle 100\rangle$ rosette, respectively.

\section{The Compton Profile}

4.1. Basic Considerations. In the so-called impulse approximation [43], the Compton profile is defined by the double 
integral in the momentum space of the ground state momentum density $\rho(\vec{p})$

$$
J_{\vec{n}}(q)=\int_{-\infty}^{\infty} \rho\left(p_{x}, p_{y}, p_{z}=q\right) d p_{x} d p_{y} .
$$

For simplicity we use a Cartesian coordinate system with the $p_{z}$ axis along the scattering direction $\vec{n} . q$ is the momentum transfer. $\rho(\vec{p})$ is the ground state momentum density defined by the sum

$$
\rho(\vec{p})=2 \sum_{n \vec{k}}|\langle\vec{p} \mid n \vec{k}\rangle|^{2} f_{n \vec{k}}
$$

extended over the first Brillouin zone and over all bands. The preceding factor is due to the spin degeneracy, $f_{n \vec{k}}$ denotes the Fermi-Dirac function. $\langle\vec{p} \mid n \vec{k}\rangle$ is the Fourier transform of the Bloch function $|n \vec{k}\rangle$

$$
|n \vec{k}\rangle=\frac{1}{(2 \pi)^{3 / 2}} \int_{V_{c}} e^{i \vec{p} \cdot \vec{r}}\langle\vec{r} \mid n \vec{k}\rangle d^{3} r,
$$

where $V_{c}$ denotes the volume of the elementary cell. As usual, the Bloch functions are normalized within the elementary cell. $\langle\vec{p} \mid n \vec{k}\rangle$ is only nonzero if $\vec{p}$ is equal to $\vec{k}$ up to a reciprocal lattice vector. The definition according equation (12) implies that the integral of EMD over the momentum space gives the number of electrons within the unit cell (EMD sum rule)

$$
\int_{\infty} \rho(\vec{p}) d^{3} p=2 \sum_{n \vec{k}} f_{n \vec{k}}
$$

Correspondingly the $\mathrm{CP}$ sum rule reads

$$
\int_{0}^{\infty} J_{\vec{n}}(q) d q=2 \sum_{n \vec{k}} f_{n \vec{k}}
$$

for any direction $\vec{n}$. Because the MAPW functions as well as their first derivatives are strictly continuous everywhere their Fourier transform asymptotically decreases at least with $1 /|\vec{p}|^{4}$ as the exact Bloch functions. The APW or KKR schemes yield $\langle\vec{p} \mid n \vec{k}\rangle$ decreasing at best with $1 /|\vec{p}|^{3}$ [44]. Nevertheless, the evaluation of the incomplete integral equation (10) needs some care even in the present case. We proceed in two steps. In Section 4.2 the influence of the spherically averaged EMD on the Compton profiles is investigated which turns out to be the dominant part. Section 4.3 deals with the directional CP's.

4.2. Contribution from the Spherically Averaged EMD. In momentum space the mean value of $\rho(\vec{p})$ over the sphere of radius $p=|\vec{p}|$ gives the spherically averaged EMD

$$
\rho_{\text {av }}(p)=\frac{1}{4 \pi} \int_{\text {sphere }|\vec{p}|=p} \rho(\vec{p}) d \omega_{\vec{p}}
$$

$d \omega_{\vec{p}}$ : unit surface element. It likewise satisfies the EMD sum rule

$$
4 \pi \int_{0}^{\infty} \rho_{\mathrm{av}}(p) p^{2} d p=2 \sum_{n \vec{k}} f_{n \vec{k}}
$$

Its contribution to the Compton profile is given by the onedimensional integral

$$
\mathrm{J}_{\mathrm{av}}(q)=2 \pi \int_{q}^{\infty} \rho_{\mathrm{av}}(p) p d p,
$$

which is evidently spherically symmetric, too. To avoid confusion with the spherically averaged Compton profile $\overline{J_{\vec{n}}(q)}$ we have used a bold font. As $\mathbf{J}_{\mathrm{av}}(q)$ also satisfies the CP sum rule

$$
\int_{0}^{\infty} \mathbf{J}_{\mathrm{av}}(q) d q=2 \sum_{n \vec{k}} f_{n \vec{k}}
$$

we expect that it is a good approximation for the spherically averaged value of $\mathrm{CP}, \overline{J_{\vec{n}}(q)}$.

Using the concept of special directions described in a previous paper [18] $\rho_{\mathrm{av}}(p)$ is directly evaluated from the EMD along 48 directions in the irreducible wedge which are defined by requiring that 111 Kubic harmonics $K_{L}\left(\vec{p}^{0}\right)$ with $l \leq 68$ have nonvanishing residua in the sense as required by Bansil [14-17]. For any direction $\vec{p}^{0}$ the contributions from the valence electrons are evaluated on a fine mesh up to 129.0 a.u. by use of MAPW results where for each value of $\vec{p}$ a Bloch vector $\vec{k}$ is found by subtracting a suitably chosen reciprocal vector $\vec{K}$, for example, $\vec{p}=\vec{k}+\vec{K}$. With the proper weights of the 48 directions formula the spherical average $\rho_{\text {av }}$ and by use of (17) $\mathbf{J}_{\mathrm{av}}(q)$ are obtained. In contrast to the free electron metals $\mathrm{Li}$ [13] and $\mathrm{Al}[12], \rho_{\mathrm{av}}$ has no discontinuities due to possible Fermi breaks. Figure 1 shows $\mathbf{J}_{\mathrm{av}}(q)$ augmented by the core profile on a semilogarithmic scale up to $q=$ 129.0 a.u. In accordance with the general considerations on the continuity of the MAPW functions [23] the contribution from the valence electrons decays by 9 orders of magnitude in the momentum range up to 129 a.u. It is expected that valence profiles obtained by KKR or FLAPW show a weaker decay. A reliable test of the numerical accuracy is the CP sum rule which gives the value 11.002 in the momentum range considered and for the $[\mathrm{Ar}]$ core. The corresponding value in the case of the $[\mathrm{Ne}]$ core is 19.002. In Figure 1 the dasheddotted curve is the Compton profile in LDA simply obtained from the SCF-MAPW density by use of

$$
J_{\mathrm{LDA}}(q)=2 \int_{V_{C}} \rho(\vec{r}) j_{i}(\rho(\vec{r}) ; q) d^{3} r .
$$

$j_{i}\left(\rho_{0} ; q\right)$ is the CP per electron of an interacting electron gas of density $\rho_{0}$. As for case Li [13] and Al [12] it roughly coincides on the semilogarithmic scale with the all electron $\mathrm{CP}$ in the case of $\mathrm{Cu}$ up to $q=12$ a.u. Again we find confirmed that the LP corrections which are based on the same functional $j_{i}$ apply to the all-electron profiles.

The Compton profiles of the valence electrons along the three principal directions have been obtained by Sakurai et al. [7] by subtracting the KKR core contributions from the measured values. Using the weights quoted in Section 2 of the previous work [12] the spherical average of the valence profiles has been derived up to $q=8$ a.u. It is remarkable that KKR and the present MAPW core contributions do 


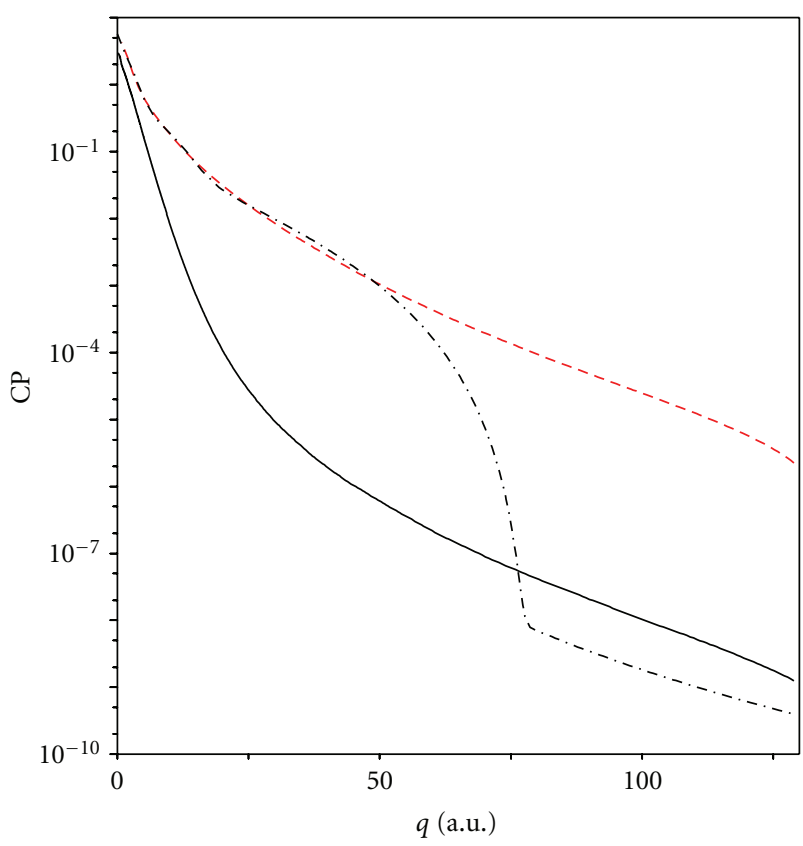

Figure 1: $\mathrm{J}_{\mathrm{av}}(q)$ of $\mathrm{Cu}$ on a semilogarithmic scale. Full curve: contribution from the valence electrons only, and dashed curve: all electron CP, dashed-dotted curve: CP in LDA using (19). In units of electrons/Bohr.

not differ significantly from the results of Biggs et al. [45] based on Hartree-Fock calculations. In Figure 13 of the previous investigations [18], theoretical profiles defined by (12) are displayed after having been folded with a Gaussian of FWHM $=0.12$ a.u. to reflect the experimental resolution. The overall shapes of both profiles are quite similar. To make this more clear in Figure 2 the differences between the theoretical, either corrected according Lam Platzman or not, and the measured profiles are plotted. The uncorrected results, displayed by the broken curve behave quite similarly as the profiles derived with the KKR scheme [7]: at low momenta the difference amounts almost up to 0.28 , after a crossover around 1.0 a.u. the deviations reach -0.07 and approach the value 0.02 near $8.0 \mathrm{a}$.u, all in electrons/Bohr. The weights just mentioned were also used to evaluate another spherical average from the CPs along the principal directions considered in details in Section 4.3. In the low momentum region it runs up to 0.033 electrons/Bohr below the broken curve. A distinct step in the direction of the experimental results is due to the LP corrections using the momentum density function of the interacting free electron gas either as proposed by Farid et al. [46] or by Ortiz and Ballone [47]. At small values of $q$ we observe a reduction by roughly 0.05 and near $q=1.0$ a.u. an increase by 0.02 electrons/Bohr. Beyond 2.0 a.u. these corrections are almost negligible. Different from the cases of $\mathrm{Li}[13]$ and $\mathrm{Al}$ [12], they are smaller but not negligible as assumed in [7] and both parameterizations produce similar corrections. The results displayed in Figure 2 are obtained within the LDA and the $[\mathrm{Ne}]$-core but the other cases behave similarly, within the

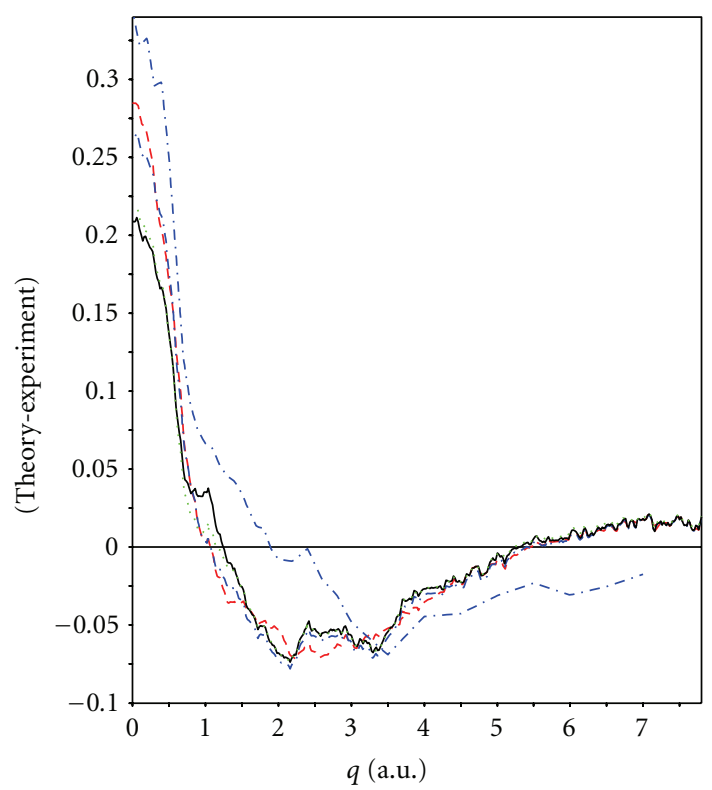

FIGURE 2: Differences between the calculated and the experimental spherically averaged valence electron Compton profiles. Full curve: LP corrections according to [46] included, dotted curve: LP corrections according to [47] included, broken curve: LP corrections excluded, dashed-dotted curve: spherical average of the CPs along the principal directions, and long-broken curve: APW result [48]. CPs in electrons/Bohr.

plotting accuracy. More quantitative insights give Table 6 which lists the deviations $\delta J$ at $q=0$ and the square root of the variance defined by

$$
\sigma=\sqrt{\int_{0}^{7.85}\left(\mathrm{~J}_{\mathrm{av}}(q)-J_{\mathrm{av}, \exp }(q)\right)^{2} d q}
$$

for each model and for both LP parameterizations. As for the form factors the Compton profiles turn out to be only slightly influenced by details of the model, for example, LDA, GGA, or the choice of the core, certainly as consequence of the CP sum rule. The $[\mathrm{Ne}]$-vwn model best competes with all others in the long-wave limit of $J(q)$, whereas in the overall agreement characterized by the variance the $[\mathrm{Ne}]$ pw91 model is the favorite. In all cases the momentum density proposed by Farid et al. [46] give a better agreement. Once more we want to emphasize that the preceding results have been obtained without any truncation, nevertheless they are considerably less CPU-time expensive than the investigations in the following section. But they fully reflect the essential trends how the CPs depend on different models and assumptions.

4.3. Directional Compton Profiles. As in a previous investigation [11] we make use of the fact that outside a circle with sufficiently large radius $P_{\max }$ the momentum density $\rho(\vec{p})$ is in a good approximation spherical with the consequence that the contribution from outside the circle is defined by

$$
\Delta J_{\vec{n}}(q)=2 \pi \int_{\sqrt{q^{2}+P_{\max }^{2}}}^{\infty} \rho(t) d t=\mathrm{J}_{\mathrm{Jav}}\left(\sqrt{q^{2}+P_{\max }^{2}}\right)
$$


TABle 6: Deviations between theoretical and experimental CPs, $\delta J$, at $q=0$ and the square root of the variance evaluated in the range $0 \leq q \leq 7.85$ a.u. These values have to be compared with the profile integrated over the same range amounting 10.896 a.u. Lower index $F$ and $O$ parameterizations according to Farid et al. [46] and Ortiz and Ballone [47], respectively. All in units electrons/Bohr.

\begin{tabular}{lcccccc}
\hline & {$[$ Ar] $]$ vwn } & {$[\mathrm{Ne}] \mathrm{vwn}$} & {$[$ Ar]pbe } & {$[\mathrm{Ne}] \mathrm{pbe}$} & {$[\mathrm{Ar}] \mathrm{pw91}$} & {$[\mathrm{Ne}] \mathrm{pw91}$} \\
\hline$\delta J$ & 0.2931 & 0.2948 & 0.2928 & 0.2977 & 0.2956 & 0.3004 \\
$\delta J_{F}$ & 0.2385 & 0.2302 & 0.2982 & 0.2433 & 0.2410 & 0.2481 \\
$\delta J_{O}$ & 0.2457 & 0.2371 & 0.2454 & 0.2812 & 0.2819 & 0.2532 \\
$\sigma$ & 0.2805 & 0.2935 & 0.2784 & 0.2315 & 0.2320 & 0.2845 \\
$\sigma_{F}$ & 0.2308 & 0.2417 & 0.2287 & 0.2335 & 0.2340 & 0.2347 \\
$\sigma_{O}$ & 0.2328 & 0.2441 & 0.2308 & & 0.2367 \\
\hline
\end{tabular}

with the $\mathbf{J}_{\mathrm{av}}$ shown in Figure 1. This procedure only produces a truncation error from the nonspherical contribution of the EMD which is roughly by the factor 10 smaller than the mean value. This consideration also allows a rough estimate of the accuracy of the KKRcalculations [7] which are based on the EMD evaluated on a mesh generated by 2421 reciprocal lattice vectors extending to about 13.0 a.u., an upper limit markedly different from 20.0 quoted by these authors. (In the fcc reciprocal lattice the last shell includes 2421 reciprocal lattice vectors of the type $(10,6,6)$ yielding a $\vec{p}$ vector of

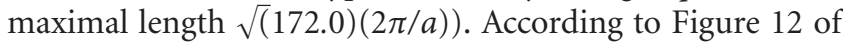
the previous paper [18] the neglected contribution $\Delta J(q)$ amounts to 0.08 and 0.06 electrons/Bohr for $q=0$ and $q=$ 8.0 a.u., respectively. From this estimate follows that Sakurai et al. [7] have considerably overestimated their accuracy by claiming that the final Compton profiles are accurate to a few parts in $10^{4}$.

In our investigation we have chosen $P_{\max }=22.5$ a.u., 26.0 a.u. and 21.8 a.u., for the direction $\vec{n}$ along [100], [110] and [111], respectively and have determined $\Delta J$ by spline interpolation of $\mathbf{J}_{\mathrm{av}}$. The evaluation of the contribution to the integral equation (21) is quite tedious since for each value of $\vec{p}$ the corresponding Bloch function $\mid n \vec{k}>$ with $\vec{k}+\vec{K}$ is needed. A considerable reduction of the numerical work is possible by use of a two-dimensional grid of points originally proposed to perform Brillouin zone integrals and which have been already used in Section 2. As shown in [6] this partition of the $\vec{p}$ space implies discrete values of $q$ defined by $(1 / 2 \mathrm{MG})(2 \pi / a) q_{[i]} I$ with the integer $I=0,1,2$ and $q_{[100]}=1, q_{[100]}=1 / \sqrt{2}$, and $q_{[111]}=1 / \sqrt{3}$. To comply with the experiments [7] and the aim to reliably identify fine structures in the profiles we have chosen $M G=40$. This gives $5740 \vec{p}$ points in the irreducible wedge of the BZ and more than 870 million points lying within the sphere of radius $P_{\max }$ in contrast to the $48 \times 1183 \times 2421$ points or to the $48 \times 505 \times 3143$ points considered in [7] or in [37], respectively. Finally the integration over the two-dimensional grid is approximated by a scheme [23] similar to that proposed by Gilat and Raubenheimer $[49,50]$ which allows to take into account the exact position of the Fermi break within the corresponding parallelepipeds. To avoid spurious contributions, the extensions of these parallelepipeds in $p_{z}$ are chosen small compared to $(1 / \mathrm{MG})(2 \pi / a)$.

The Compton profiles of the valence electrons along the principal directions are displayed in Figure 3. LP corrections have been included in the full curve. Both theoretical curves are convoluted with a Gaussian with full width at half maximum (FWHM) of 0.12 a.u. according to Sakurai et al. [7]. For comparison the corresponding measured CPs are plotted which have been renormalized in [111]-direction by $0.438 \%$ according to the results [Ne]vwn with LP corrections included. Obviously the LP corrections slightly improve the agreement with the experimental results. But they still remain higher than the measured ones up to $q=$ 0.75 a.u. and lie slightly below them in the momentum range [1.32-5.0] a.u. Both theoretical profiles show a weak wiggly structure in the [110] profile.

Figure 4 showing the differences between the theoretical and measured profiles along the principal directions makes the deviations more clear. The uncorrected results shown in panel (a) behave quite similarly to those derived with the KKR-scheme by Sakurai et al. [7]. As far as comparison is possible the MAPW calculations yield a slightly larger deviation near $q=0$. According to the estimate at the beginning of this section this is a consequence of the smaller value of the cut-off $P_{\max }$ used in the KKR investigations [7]. The LP-corrections based on the momentum density functions $[46,47]$ reduce the deviations in the momentum range up to $q=0.8$ a.u., especially near $q=0$ by 0.05 a.u. but have only slight influence beyond 1.5 a.u. As already found in Section 4.2 the momentum density of the interacting electron gas proposed by Ortiz and Ballone [47], Figure 4(b), and by Farid et al. [46], Figure 4(c), yield similar curves.

The orientation dependence of the Compton profile is quite small as shown in Figure 5 where the profile differences along the principal directions obtained by MAPW are compared with the experimental results. By this display not only the core contributions and the many-body aspects treated by the LP procedure but also the asymptotic tail of the momentum density in the integrand of (21) are completely absent in the theoretical curves since all these contributions only depend on the absolute magnitude of the momentum $\vec{q}$. Our results are quite similar to those of the previous investigations of the author [6] and the KKR work [7] and show that the anisotropy of the CPs is only slightly influenced by the band structure schemes and details of the potential. The agreement with the experimental results is satisfactory. In the both differences $\Delta J_{[111]-[110]}$ and $\Delta J_{[110]-[100]}$ not only the periodicity of the oscillations up to $p=4.0$ a.u. but also the locations of some fine structures are properly described. The heights of the oscillations are distinctly overestimated. 


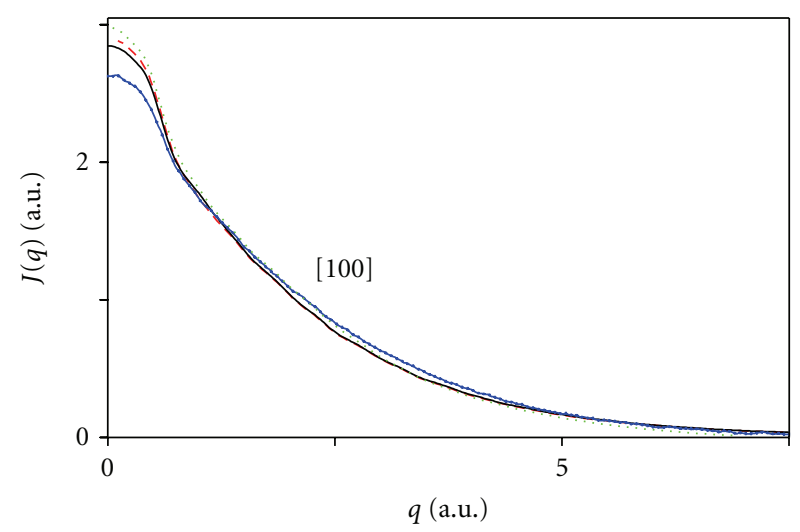

(a)

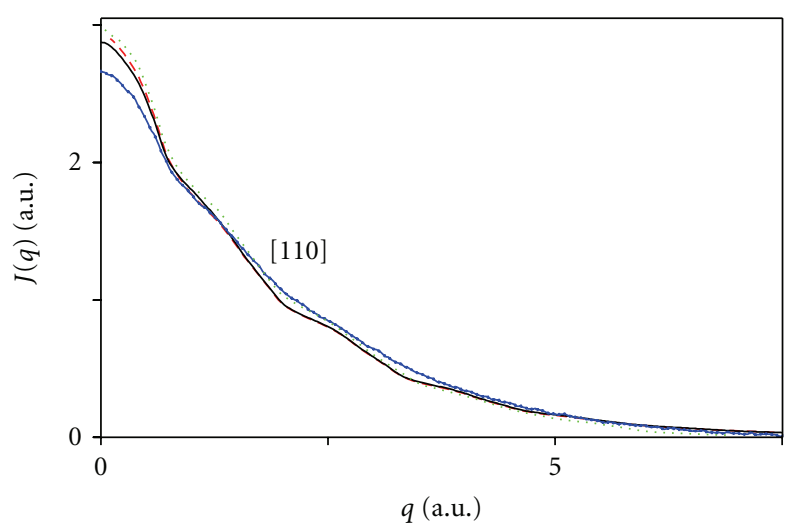

(b)

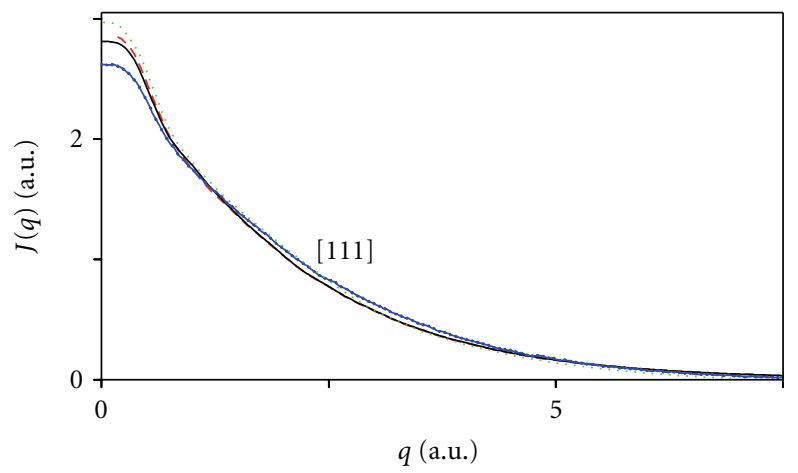

(c)

FIgURE 3: The valence electron Compton profiles along [100,110] and [111] obtained by subtracting all core contributions. Full curves: theoretical result with LP corrections included, dashed curves without LP corrections; both convoluted with a Gaussian with FWHM of 0.12 a.u. Crosses connected by thin curves: experimental results [7]. Dotted curves: APW results [48].

The broken curve in Figure 5 is obtained by use of the Fourier-Hankel method [51] based on the expansion of the EMD in Kubic harmonics $K_{l}$ in the present case up to $l=28$. With the notation of [13] Section 4 we get, for example,

$$
\Delta J_{[110]-[100]}(q)=2 \pi \sum_{L}\left(F_{[110], L}-F_{[100], L}\right) g_{L}(q) .
$$

Because both $\left(F_{[110], L}\right.$ and $\left.F_{[100], L}\right) g_{L}$ are defined by azimuthal integrals over Kubic Harmonics, see (11) of [13], their differences can be obtained without loss of accuracy. The functions $g_{L}(q)$ are found by folding the EMD expansion in $\mathrm{KHs}$ with the Legendre polynomial of the same degree. From the close agreement of both theoretical curve we learn that the Fourier-Hankel method, which is considerably less CPU demanding than the two-dimensional integration according to equation (21), is also well suited for the evaluation of the directional profiles $J_{\vec{n}}$ provided the momentum density $\rho(\vec{p})$ is known along a sufficiently dense grid of orientations $\vec{p}^{0}$.

The second derivatives of the theoretical and experimental profiles $d^{2} J(q) / d q^{2}$ derived by spline interpolation and convoluted with a Gaussian of FWHM $=0.12$ a.u. show good overall agreement as to both the positions and the heights of the peaks. However, the distinct maxima occurring in [100] and [110] directions cannot be assigned to any Fermi diameter. In contrast to $\mathrm{Li}$ and $\mathrm{Al}$, measurements of the Compton profiles are not suited to map out the Fermi surface in the case of $\mathrm{Cu}$.

4.4. Another Explanation of the Disagreement Near $q=0$. Measured profiles are mostly corrected by computer simulation of the multiple Compton scattering events which need specific assumptions for the scattering process. Different from Sakurai et al. [7], Bauer and Schneider [52] used a semiempirical approach which is based on the theoretical valence electron $\mathrm{CP}$ derived by using a basis of Gaussian orbitals [53]. It is justified by the fact that the corrected profiles for samples of different thicknesses coincide within a margin of 0.03 a.u. Figure 6 shows the profiles for the [110] direction after being corrected compared with the theoretical profile. Both profiles mostly differ by less than 0.02 a.u. over the whole momentum range up to 5.0 a.u. Near $q=0$ the discrepancy is completely reduced making other explanations obsolete.

4.5. A Comparison with the KKR Investigations [7]. Apart from the choice of some intrinsic parameters as already mentioned the KKR investigations [7] are essentially different from the present one in various points. (i) They are based on the muffin-tin approximations with the consequence that the wave functions outside the APW spheres are not variationally given and, therefore, are less accurate than those obtained 


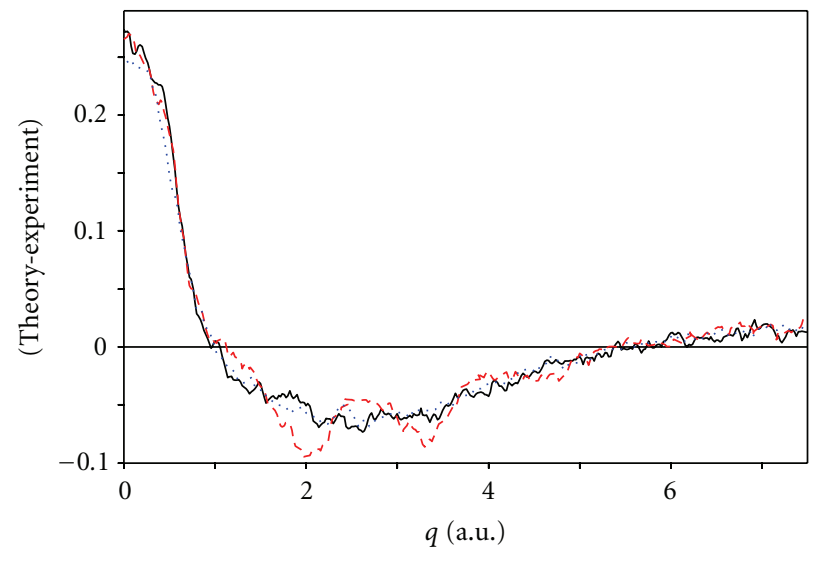

(a)

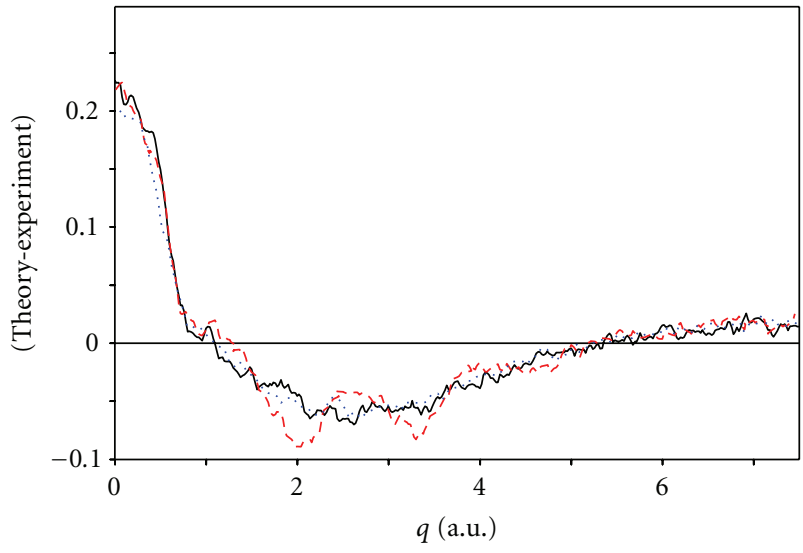

(b)

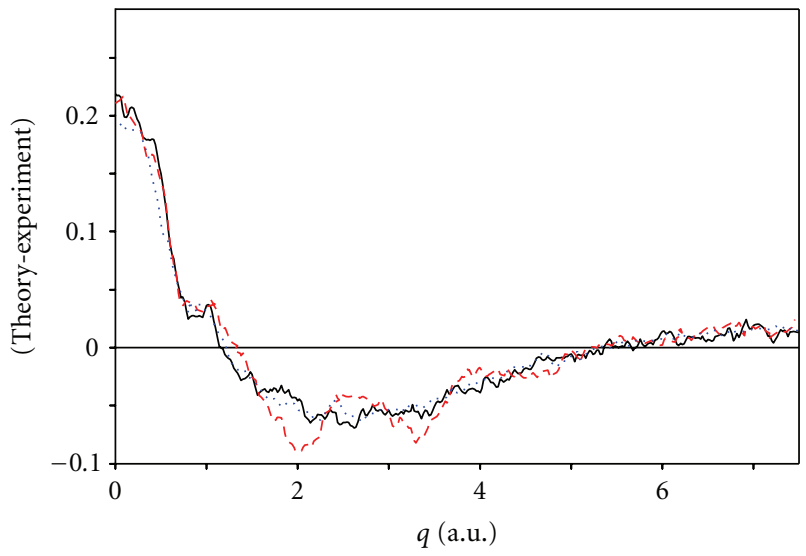

(c)

FIGURE 4: Differences between the calculated and the experimental valence-electron Compton profiles. (a) LDA results without LP corrections, (b) LP corrections [47] included, (c) LP corrections [46] included. [100]: full curve, [110]: dashed curve, [111]: dotted curve. All in units of electrons/Bohr.

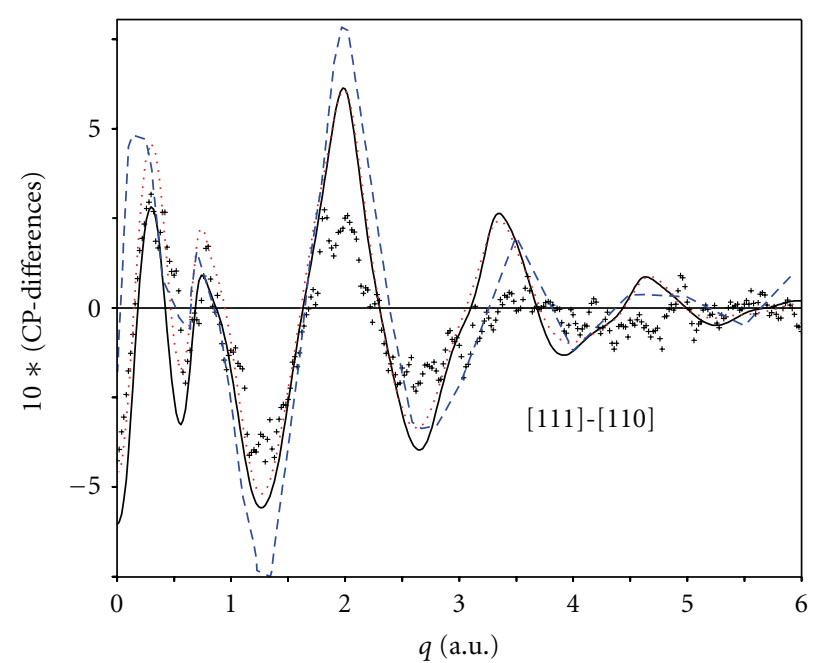

(a)

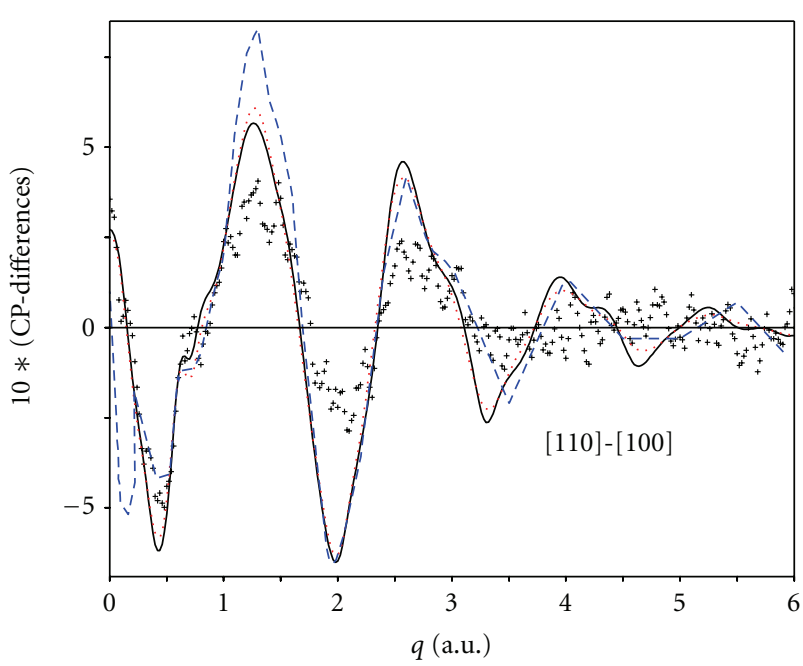

(b)

FIGURE 5: Directional Compton differences. Upper panel: [111]-[110] lower panel: [110]-[100]. Full curve: theoretical results, broken curve: Fourier-Hankel reconstruction, long-broken curve APW results [48] string of crosses: experimental data. All in units of electrons/Bohr. 


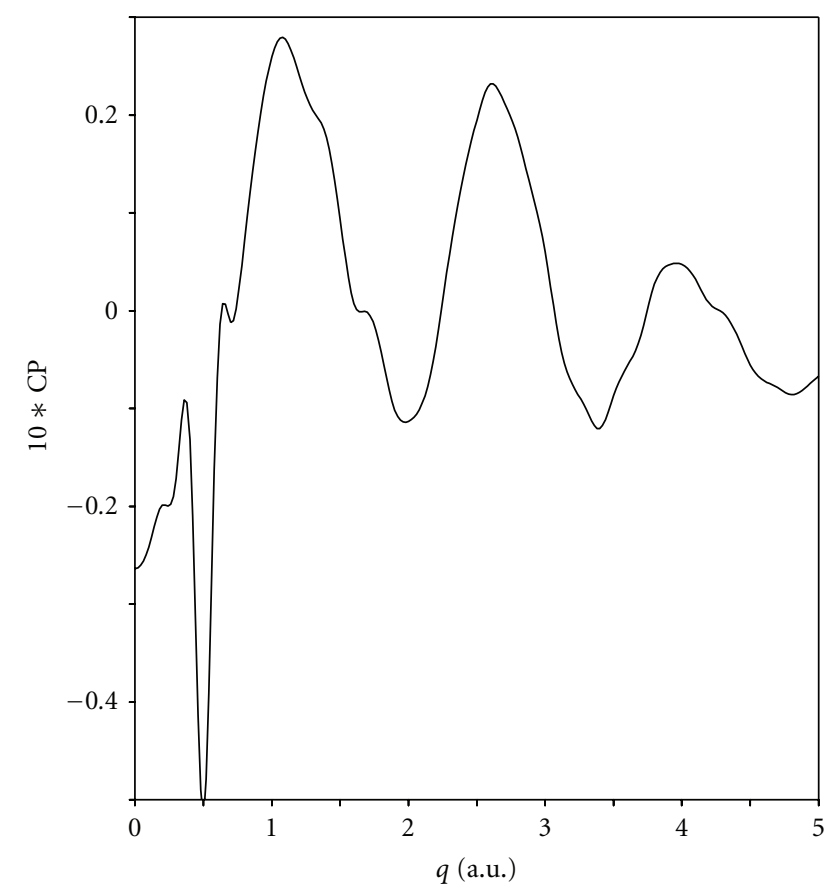

Figure 6: Difference between experimental [52] and theoretical all-electron profiles along the [110] directions. All in units of electrons/Bohr, profile difference enlarged by a factor of 10 .

by MAPW. (ii) Less sophisticated exchange and correlation functionals [54] are used in the SCF scheme and in the evaluation of the Lam-Platzman corrections. (iii) No details are given how the latter are determined, especially outside the APW-spheres. (iv) The Compton profiles are evaluated by an improper integral which has been truncated at a rather small value of $|\vec{p}|$. (v) In contrast to a generalizations of the Gilat-Raubenheimer scheme which saves considerably computer time [55] the tetrahedron method has been used for the evaluation of the momentum density. Despite these differences with respect to methodology and computational accuracy the overall similarity of the profiles obtained by completely different schemes is remarkable. In the case of the noble metal $\mathrm{Cu}$ investigations of the Compton profiles are no proper mean for a decision in favour of one theoretical scheme.

\section{Conclusions}

The preceding considerations had the result that there exists no unique approach which gives the best agreement with the experimental facts. LDA with the $[\mathrm{Ne}]$-core describes the energetics and the form factors considerably well whereas the Compton profiles are slightly favoured by the GGA functionals proposed by Perdew et al. [4] and the [Ne] core. In a consistent treatment the former approach requiring a larger set of variational functions is to be preferred. This dilemma is certainly due to many-body effects not properly taken into account. Undoubtedly, those models and approximations should be favoured which explain simultaneously different phenomena sufficiently well. The full-potential linearized
APW investigations by Kubo et al. [37] do not meet with these criteria as they show a quite good agreement with the measured profiles [7] when self-interaction corrections are taken into account, as in the case of free atoms [56], but on the other hand, these corrections change the band structure, especially near the Fermi level, in a rather unconventional way.

Our hope that one of the approaches will essentially reduce the disagreement of the profiles near $q=0$ that has not been fulfilled. The present investigations rule out that the treatment of the core contributions is the origin of the disagreement: as mentioned the core contributions evaluated by MAPW differ from Biggs atomic profiles by less than $1 \%$. Nevertheless, the experimentalists are recommended to report in future also the core $\mathrm{CP}$ which they use to shift their data. Thus it remains an open question where the origin of the discrepancy lies. It may either be caused by the handling of the measured data or by many-body effects which are not taken into account by the Lam Platzman corrections.

\section{Acknowledgments}

The author would like to thank Professor Y. Sakurai for providing results of their high-resolution Compton-scattering study. Dr. Herbert Stöhr has accompanied these and the preceding investigations with great engagement. For this support and the critical reading of the paper many thanks are due. The help of Dr. Reinhold Bader as well as the generous hospitality of Professor Jan von Delft and Professor U. Schollwöck are gratefully acknowledged.

\section{References}

[1] H. Bross, "Verallgemeinerung der MAPW-Methode für ein beliebiges Potential," Helvetica Physica Acta, vol. 41, p. 717, 1968.

[2] H. Bross, G. Bohn, G. Meister, W. Schubö, and H. Stöhr, "New version of the modified augmented-plane-wave method," Physical Review B, vol. 2, no. 8, pp. 3098-3103, 1970.

[3] S. H. Vosko, L. Wilk, and M. Nusair, "Accurate spin-dependent electron liquid correlation energies for local spin density calculations: a critical analysis," Canadian Journal of Physics, vol. 58, no. 8, pp. 1200-1211, 1980.

[4] J. P. Perdew, J. A. Chevary, S. H. Vosko et al., "Atoms, molecules, solids, and surfaces: applications of the generalized gradient approximation for exchange and correlation," Physical Review B, vol. 46, no. 11, pp. 6671-6687, 1992.

[5] J. P. Perdew, K. Burke, and M. Ernzerhof, "Generalized gradient approximation made simple," Physical Review Letters, vol. 77, no. 18, pp. 3865-3868, 1996.

[6] H. Bross, "Band structure calculation of the momentum density and Compton profile of copper," Journal of Physics F, vol. 12, no. 10, pp. 2249-2266, 1982.

[7] Y. Sakurai, S. Kaprzyk, A. Bansil et al., "High-resolution Compton scattering study of $\mathrm{Cu}$ : experiment and theory," Journal of Physics and Chemistry of Solids, vol. 60, no. 7, pp. 905-910, 1999.

[8] J. C. Slater, "Wave functions in a periodic potential," Physical Review, vol. 51, no. 10, pp. 846-851, 1937.

[9] H Bross, "Electronic structure of the cubic compounds ReGa $(\mathrm{Re}=\mathrm{Er}, \mathrm{Tm}, \mathrm{Yb}$, and Lu)," Advances in Condensed Matter Physics, vol. 2011, Article ID 867074, 14 pages, 2011. 
[10] H. Bross, "Investigation of some ground state properties of lithium with the all electron MAPW method," Physica Status Solidi B, vol. 229, no. 3, pp. 1359-1370, 2002.

[11] H. Bross, "LDA and GGA investigations of some ground state properties of aluminium with the all electron MAPW method," European Physical Journal B, vol. 37, no. 3, pp. 405411, 2004.

[12] H. Bross, "The local density approximation limit of the momentum density and the Compton profiles of Al," Journal of Physics Condensed Matter, vol. 16, no. 41, pp. 7363-7378, 2004.

[13] H. Bross, "Electronic structure of $\mathrm{Li}$ with emphasis on the momentum density and the Compton profile," Physical Review B, vol. 72, no. 11, Article ID 115109, 14 pages, 2005.

[14] A. Bansil, "Special directions in the brillouin zone," Solid State Communications, vol. 16, no. 7, pp. 885-889, 1975.

[15] W. R. Fehlner and S. H. Vosko, "Product representation for cubic harmonics and special directions for the determination of the fermi surface and related properties," Canadian Journal of Physics, vol. 54, no. 21, pp. 2159-2169, 1976.

[16] W. R. Fehlner, S. B. Nickerson, and S. H. Vosko, "Cubic harmonic expansions using gauss integration formulas," Solid State Communications, vol. 19, no. 1, pp. 83-86, 1976.

[17] R. Prasad and A. Bansil, "Special directions for Brillouin-zone integration: application to density-of-states calculations," Physical Review B, vol. 21, no. 2, pp. 496-503, 1980.

[18] H. Bross, "Special directions for surface integrals in cubic lattices with application to the evaluation of the Compton profile of copper," Physica Status Solidi B, vol. 243, no. 3, pp. 653-665, 2006.

[19] L. Lam and P. M. Platzman, "Momentum density and Compton profile of the inhomogeneous interacting electronic system. I. Formalism," Physical Review B, vol. 9, no. 12, pp. 5122-5127, 1974.

[20] A. Baldereschi, "Mean-value point in the Brillouin zone," Physical Review B, vol. 7, no. 12, pp. 5212-5215, 1973.

[21] D. J. Chadi and M. L. Cohen, "Special points in the brillouin zone," Physical Review B, vol. 8, no. 12, pp. 5747-5753, 1973.

[22] H. J. Monkhorst and J. D. Pack, "Special points for Brillouinzone integrations," Physical Review B, vol. 13, no. 12, pp. 5188 5192, 1976.

[23] H. Bross, "Pseudopotential theory of the dielectric function of Al-the volume plasmon dispersion," Journal of Physics F, vol. 8, no. 12, pp. 2631-2649, 1978.

[24] J. P. Perdew, "Density-functional approximation for the correlation energy of the inhomogeneous electron gas," Physical Review B, vol. 33, no. 12, pp. 8822-8824, 1986.

[25] J. P. Perdew, "Density-functional approximation for the correlation energy of the inhomogeneous electron gas," Physical Review B, vol. 34, no. 10, p. 7406, 1986.

[26] F. D. Murnaghan, "The compressibility of media under extreme pressures," Proceedings of the National Academy of Sciences of the United State, vol. 30, no. 9, pp. 244-247, 1944.

[27] U. Himmler, H. Peisl, A. Sepp, and W. Waidelich, "Ein Verdampfungskyrostat für Präzisions-Röntgenstreuuntersuchungen zwischn $4^{\circ} \mathrm{K}$ und $350^{\circ} \mathrm{K}$," Zeitschrift für Angewandte Physik, vol. 28, p. 104, 1969.

[28] S. Ajit, K.-H. Bhalla, H. L. Hellwege et al., Landolt-Börnstein, vol. 6, Springer, Berlin, Germany, 1971.

[29] W. C. Overton and J. Gaffney, "Temperature variation of the elastic constants of cubic elements. I. copper," Physical Review, vol. 98, no. 4, pp. 969-977, 1955.
[30] A. Seeger and O. Buck, "Die experimentalle Ermittelung der elastischen konstanten hoherer Ordnung," Zeitschrift Naturforschung A, vol. 15, pp. 1056-1067, 1960.

[31] V. L. Moruzzi, J. F. Janak, and A. R. Williams, Calculated Electronic Properties of Metals, Pergamon Press, New York, NY, USA, 1978.

[32] S. Kurth, J. P. Perdew, and P. Blaha, "Molecular and solid-state tests of density functional approximations: LSD, GGAs, and Meta-GGAs," International Journal of Quantum Chemistry, vol. 75, no. 4-5, pp. 889-909, 1999.

[33] S. Ajit, K.-H. Bhalla, H. L. Hellwege et al., Landolt-Börnstein, vol. 13c, Springer, Berlin, Germany, 1984.

[34] P. T. Coleridge and I. M. Templeton, "High precision de Haasvan Alphen measurements in the noble metals," Journal of Physics F, vol. 2, no. 4, pp. 643-656, 1972.

[35] I. M. Templeton, "The effect of hydrostatic pressure on the fermi surfaces of copper, silver, and gold. II. High precision studies," Canadian Journal of Physics, vol. 52, no. 17, pp. 16281634, 1974.

[36] J. Laverock, T. D. Haynes, M. A. Alam, and S. B. Dugdale, "Experimental determination of the state-dependent enhancement of the electron-positron momentum density in solids," Physical Review B, vol. 82, no. 12, Article ID 125127, 2010.

[37] Y. Kubo, Y. Sakurai, and N. Shiotani, "Effects of selfinteraction correction on momentum density in copper," Journal of Physics Condensed Matter, vol. 11, no. 7, pp. 16831695, 1999.

[38] M. R. Halse, "The fermi surfaces of the noble metals," Philosophical Transactions of the Royal Society A, vol. 265, no. 1167, pp. 507-532, 1969.

[39] S. Ajit, K.-H. Bhalla, H. L. Hellwege et al., Landolt-Börnstein, vol. 13c, Springer, Berlin, Germany, 1984.

[40] P. T. Coleridge and I. M. Templeton, "Fermi-surface radii in copper, silver, and gold," Physical Review B, vol. 25, no. 12, pp. 7818-7819, 1982.

[41] J. R. Schneider, N. K. Hansen, and H. Kretschmer, "A charge density study of copper by $\gamma$-ray diffractometry on imperfect single crystals," Acta Crystallographica A, vol. 37, pp. 711-722, 1981.

[42] R. J. Temkin, V. E. Henrich, and P. M. Raccah, "Experimental charge density of copper," Physical Review B, vol. 6, no. 10, pp. 3572-3581, 1972.

[43] P. Eisenberger and W. A. Reed, "Relationship of the relativistic Compton cross section to the electron's velocity distribution," Physical Review B, vol. 9, no. 8, pp. 3237-3241, 1974.

[44] H. Roth-Seefrid and H. Bross, "Asymptotic behaviour of the Fourier coefficients of MAPW wave functions," Zeitschrift für Physik B, vol. 26, no. 2, pp. 125-131, 1977.

[45] F. Biggs, L. B. Mendelsohn, and J. B. Mann, "Hartree fock Compton profiles for the elements," Atomic Data and Nuclear Data Tables, vol. 16, no. 3, pp. 201-309, 1975.

[46] B. Farid, V. Heine, G. E. Engel, and I. J. Robertson, "Extremal properties of the Harris-Foulkes functional and an improved screening calculation for the electron gas," Physical Review B, vol. 48, no. 16, pp. 11602-11621, 1993.

[47] G. Ortiz and P. Ballone, "Correlation energy, structure factor, radial distribution function, and momentum distribution of the spin-polarized uniform electron gas," Physical Review B, vol. 50, no. 3, pp. 1391-1405, 1994.

[48] P. Papanicolaou, N. C. Bacalis, and D. A. Papaconstantopoulus, Handbook of Calculated Electron Momentum Distributions, Compton Profiles, and Xray Form Factors of Elemental Solids, CRC Press, Boca Raton, Fla, USA, 1991. 
[49] G. Gilat and L. J. Raubenheimer, "Accurate numerical method for calculating frequency-distribution functions in solids," Physical Review, vol. 144, no. 2, pp. 390-395, 1966.

[50] L. J. Raubenheimer and G. Gilat, "Accurate numerical method of calculating frequency distribution functions in solids. II. Extension to hcp crystals," Physical Review, vol. 157, no. 3, pp. 586-599, 1967.

[51] P. E. Mijnarends, "Determination of anisotropic momentum distributions in positron annihilation," Physical Review, vol. 160, no. 3, pp. 512-519, 1967.

[52] G. E. W. Bauer and J. R. Schneider, "Electron correlation effect in the momentum density of copper metal," Physical Review B, vol. 31, no. 2, pp. 681-692, 1985.

[53] D. Bagayoko, D. G. Laurent, S. P. Singhal, and J. Callaway, "Band structure, optical properties, and compton profile of copper," Physics Letters A, vol. 76, no. 2, pp. 187-190, 1980.

[54] U. von Barth and L. Hedin, "A local exchange-correlation potential for the spin polarized case. I," Journal of Physics C, vol. 5, no. 13, pp. 1629-1642, 1972.

[55] H. Bross, "On the efficiency of different schemes for the evaluation of the density of states and related properties in solids," Physica Status Solidi B, vol. 179, no. 2, pp. 429-439, 1993.

[56] N. Hamada and S. Ohnishi, "Self-interaction correction to the local-density approximation in the calculation of the energy band gaps of semiconductors based on the full-potential linearized augmented-plane-wave method," Physical Review B, vol. 34, no. 12, pp. 9042-9044, 1986. 

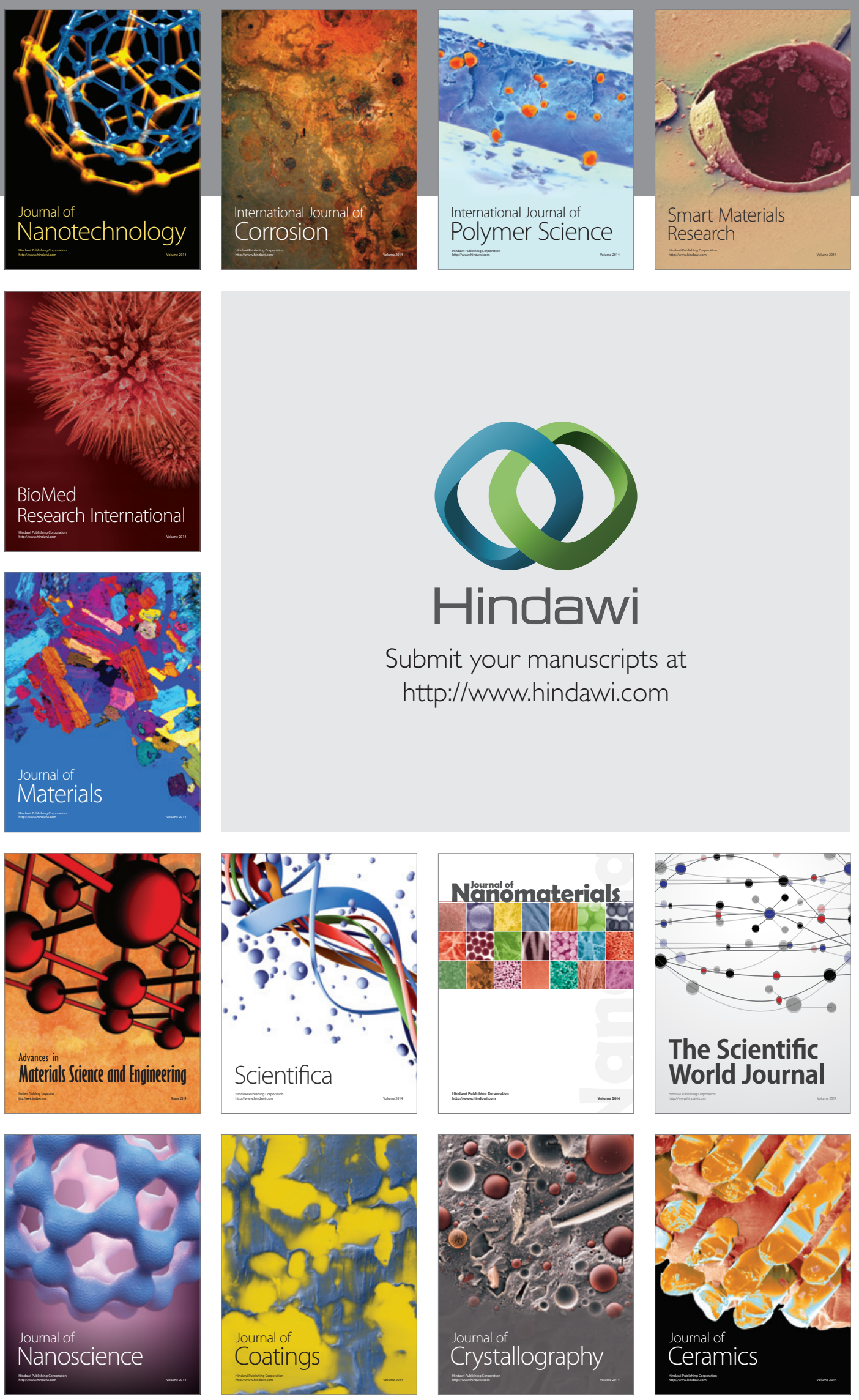

The Scientific World Journal

Submit your manuscripts at

http://www.hindawi.com

\section{World Journal}

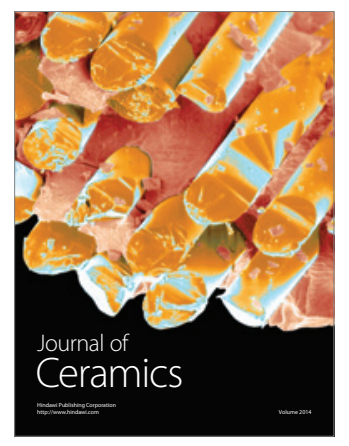

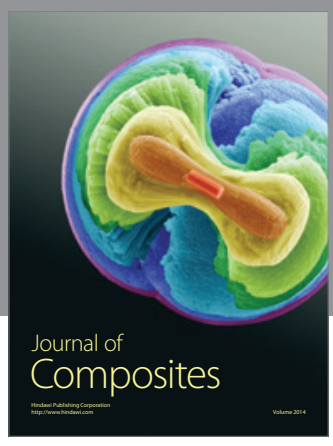
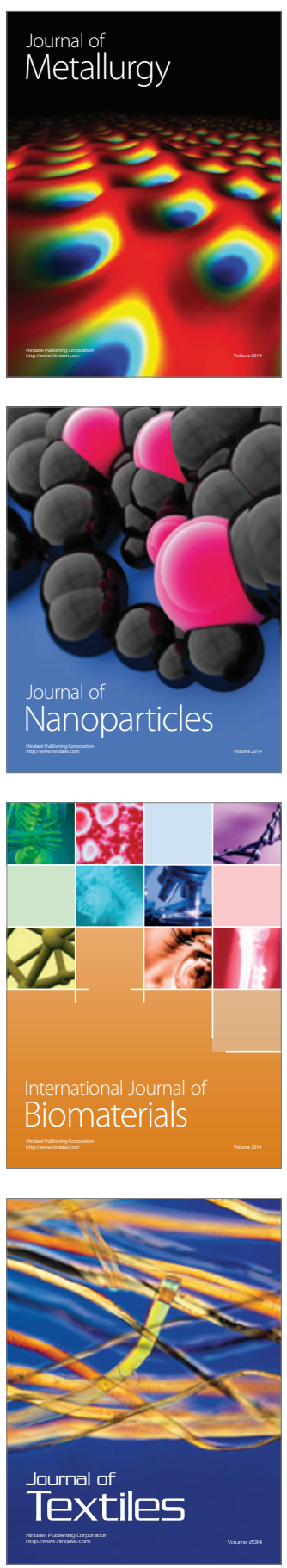